\title{
DESIGNING AUGMENTED REALITY SERVICES FOR E-BUSINESS: A PROJECT MANAGEMENT PERSPECTIVE
}

\author{
Talib Tahirović, Tamara Naumović, Lazar Živojinović, Zorica Bogdanović, \\ Marijana Despotović-Zrakić \\ Faculty of Organizational Sciences, University of Belgrade, Serbia
}

\begin{abstract}
The subject of this paper is an analysis of the concepts and application of the augmented reality in e-commerce. The literature in the field suggests a huge potential for improving ecommerce using augmented reality services. The goal of the paper is to provide a new insight into this field through an example of development of an augmented reality service for furniture manufacturing company. The project includes creation of a virtual catalog of products which consists of 3D models of products and their positioning in real space. User interaction with the developed system is performed through mobile devices. The Vuforia and Unity platform were used for the realization of the project. The project was managed using Scrum.
\end{abstract}

Key words: 3D modeling, augmented reality, e-commerce, technologies.

\section{INTRODUCTION}

The past decade has been marked by the rise of the interest and participation of users in ecommerce. Not only has there been an increase of interest, but the industry has also recorded a great increase in profit. Likewise, the development of technology creates new ways of establishing commercial trading systems (Lee, 2009).

It is long since technology was just a basis of e-commerce; nowadays it is turning the industry into what could be the foundation of modern retail business. The actual experience of shopping is one of the areas in which ecommerce has succeeded more than traditional retail businesses. Nothing could measure up to the experience of entering a shop and interacting with a product or a service, at least until now. The use of virtual reality (further: VR) and augmented reality (further: AR) technologies has made it possible for the manufacturers in e-commerce to showcase their products in great detail, which makes them comparable to their tangible versions. The development of technologies which provides us with this way of making a product more approachable to the end user redefines the shopping experience. Besides marketing, the integration of VR and AR can provide the users with the opportunity to immediately visualize themselves using the product and provide a unique way of user engagement. A simple approach to instant communication, transmission of information, logical analysis and online connecting has made it possible to remotely administer sustainable e-commerce platforms. Retailers no longer have to minimize all the aspects of their business or possess a large initial capital. (Paul, 2004)

The goal of this paper is to develop a virtual catalogue based on the technology of AR. It is necessary to analyze the existing solutions, alter them according to the requirements of the specific industry and to create an application that will fulfill the expectations of the end users. Throughout development of an actual example in the context of furniture industry, the possibilities of the usage of AR in ecommerce will be demonstrated.

\section{LITERATURE OVERVIEW}

$\mathrm{AR}$ is an interactive experience of the real world, whereby its elements are "augmented" by computer-generated perceptual information, sometimes through multiple senses, such as sight, hearing and touch. AR or supplemented reality represents the user's representation of the world broadened by computer-generated text, images and sound (Patrick, 2017). 
Broadened sensory information can be constructive (additional to the natural surroundings) or destructive (thereby masking the natural surroundings) and they seamlessly intertwine with the physical world so that it becomes the perfect version of the natural surroundings. (Global Augmented Reality (AR) Market Professional Survey Report, 2018) In this way AR changes the immediate perception of the actual surroundings, whereas VR completely replaces the user's actual reality with a simulated one. AR is related to two synonyms: a mixed reality and a computermediated reality (Tucker, Pierera, Shaw, \& Underwood, 2018).

AR services have introduced improvements in sale and production of furniture. One of the problems in application in this type of technology in the furniture industry is positioning of virtual objects in the real space, or desired part of the house or business space. (Ma, Gausemeier, Fan, \& Grafe, 2009) One of the AR categorizations regarding positioning of objects would be (Rampolla \& Kipper, 2012):

- augmented reality based on markers,

- augmented reality based on projection

- augmented reality based on parameter reading.

The primary value of the augmented reality is that it brings the components of the digital world into real world perception and this is not implemented through simple presentation of data, but through the integration of immersed sensations perceived as natural parts of an environment. (Craig, 2013)

The first commercial experiences of augmented reality were used in entertainment and games (Ma, Jain, \& Anderson, 2014). Now, other industries have also been interested in AR opportunities, for example in knowledge exchange, education, information flow management and organization of remote meetings, application of augmented reality technologies has become up-to-date (Peddie, 2017). Further, AR is used to improve the natural environment or situations and offers the enlightened experience. Using advanced AR technologies (such as adding a computer vision and identifying objects), information about the real world of users becomes interactive.
The key measurement unit of the augmented reality system is the realism and quality of integration of the expansion into the real world. The software must extract real world coordinates from the image independent of the camera. This process is called image recognition and uses different methods of computer vision and is most closely related to video monitoring. Many methods of computer vision related to the augmented reality are inherited from visual odometric (Ronald, Baillot, Behringer, Feiner, Julier, \& MacIntyre, 2001). Those methods usually contain two parts. The first is detection of points of interest or markers of authenticity in photos. The first phase can use features detection, such as recognition of corners, edges and image processing. The second phase shows coordinate system of the real world based on data obtained in the first phase. Some methods assume that objects on the scene have correct geometry. If part of the scene is unknown, SLAM simultaneous localization and mapping can be used to get relative positions. Methods of structure through motions are being used if there is no information about geometry of the scene. The mathematical methods used in the second phase include projective epipolar geometry, geometric algebra, rotation representation, exponential maps, nonlinear optimization and statistics (Craig, 2013).

AR includes hardware components such as processors, displays, sensors and input devices. Modern mobile phones, computer devices such as smartphones and tablet computers contain all elements which usually include the camera, MEMS sensors - accelerometer, GPS and compass, making them suitable for the augmented reality platform (Metz, 2012).

AR will soon change the form of trade. The main advantage for creating a business in field of AR development is that the hardware is available and its use is intuitive and understandable. In the next few years, users will be able to try out clothes without actually wearing it or check whether furniture fits into the interior (Peddie, 2017). Moreover, with technological evolution, other human sensors such as smell, touch, and feel will emerge, which will enable better usage of this type of technology. Developing an AR app for Android or iOS reveals a new experience for 
offline and online shopping. Virtual rooms help users to choose the right size of clothes or furniture for their apartment. Also, this technology can be used for promotional campaigns, adding augmented reality to materials.

Augmented reality as technological progress represents new opportunities for other markets and fields as well, including banking, real estate, education, health and production. AR can contribute and speed up the production process in a factory. Project managers can monitor real time work progress through AR markers located on equipment. In addition, time can be saved using digital maps and plans (Furht, 2011). This technology brings unlimited opportunities for teaching and learning process (Kurubacak, 2017). An additional reality provides a unique cognitive path with impressive simulations in real life. The technology provides a completely realistic psychological and physical experience and helps in obtaining an authentic virtual experience that can be realized in real life (Ariso, 2017).

Augmented reality affects all aspects of business and the biggest progress has been made in e-commerce (Radenković, Despotović-Zrakić, Bogdanović, Barać, \&
Labus, 2015). Manufacturers have the opportunity to find customers in a different way and display their products in virtual catalogs. Each product is detailed and displayed and the customer can see the product in real space and in real size. Therefore, it is possible to bring the product closer to the end user, which contributes to the previous experience in e-commerce (Jung \& Dieck, 2018).

\section{PROJECT MANAGEMENT OF DEVELOPMENT OF AR SERVICES IN FURNITURE INDUSTRY}

Designing an AR service for a furniture manufacturing company is a complex task. The service needs to be integrated with the product design phase, because 3D models of the products are created while designing the actual products. Modifications of 3D models need to be done in order to integrate these models into an AR application and fit it into the AR space (Mullen, 2011).

Project development and management is based on agile methodologies (Bogojević, 2017). Project management is done using Scrum methods. The iteration cycles in Scrum lasted for 30 days, fixed (Figure 1).

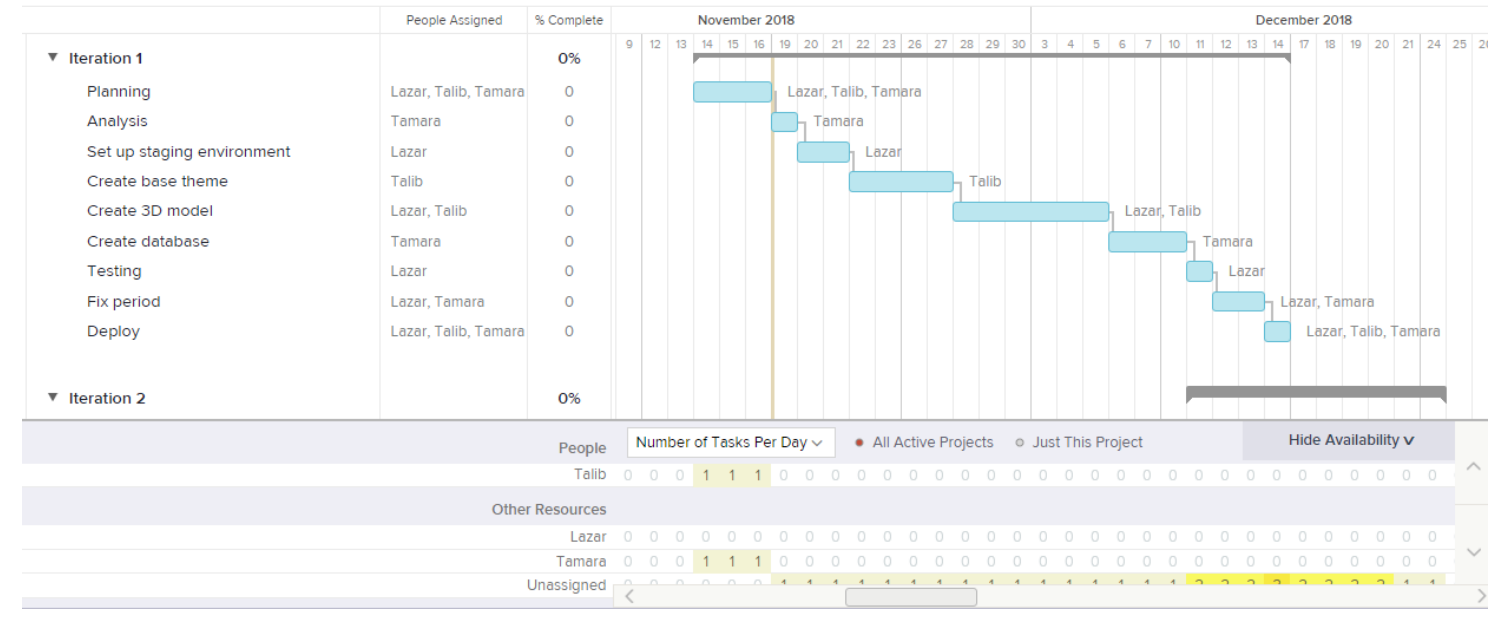

Figure 1: Scrum iteration cycles

Every iteration consists of the following:

1. Requirement analysis

It is necessary to:

- Collect and analyze user requirements which will make the foundation for the system and design of the services.
- Create a virtual catalog of products that will allow us to display a particular product in real space. 
- Adapt the already developed furniture models to the needs of the services.

- Enter basic information for each model: availability, available colors and dimensions.

- Enable the display of the products in the space, product change and ordering process.

2. Design

The design process begins with the adaptation of the environment and launching the necessary AR tools. Creating a 3D model of furniture is done using the Autodesk 3ds Max program. Each model needs to be processed in detail so it represents an identical picture of a real piece of furniture. Designing involves creating a database in Cloud where 3D models and a detailed description of the model are stored. Software development is done through Unity engine and Vuforia SDK software (Morales, 2015). The system architecture is shown in Figure 2.

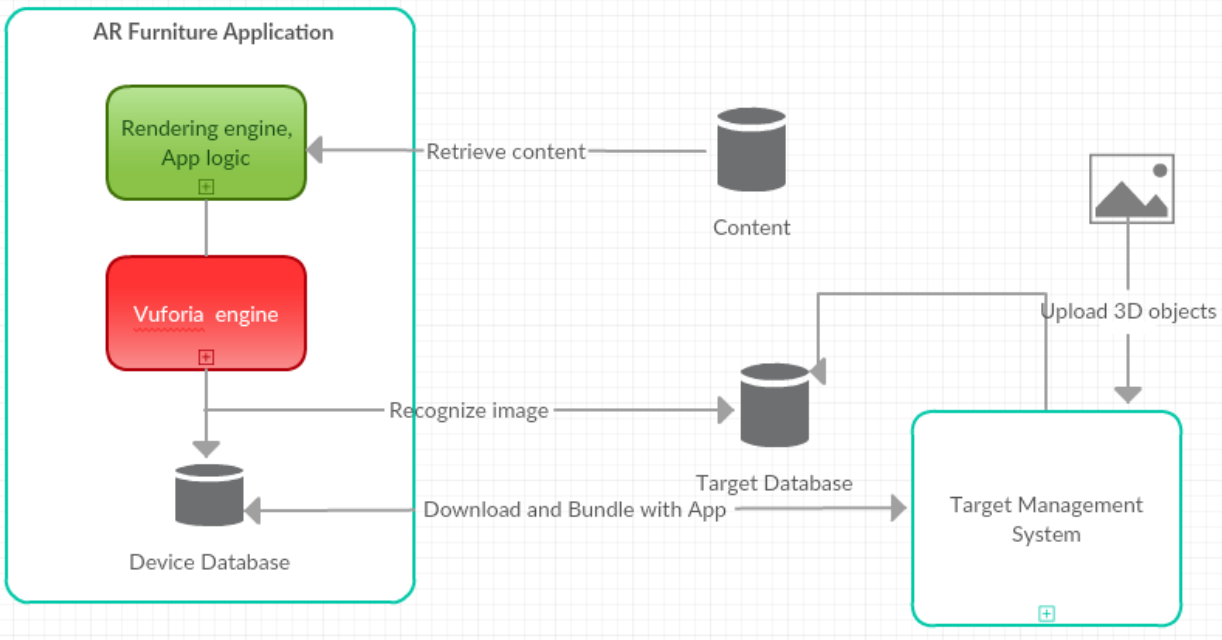

Figure 2: System architecture

The mobile device analyzes space using its camera, detects the position of the user, and determines the distance of the object in relation to the starting point. The way software recognizes a place in real space for the placement of virtual object is determined in the design process. Virtual object generation process is shown in Figure 3.

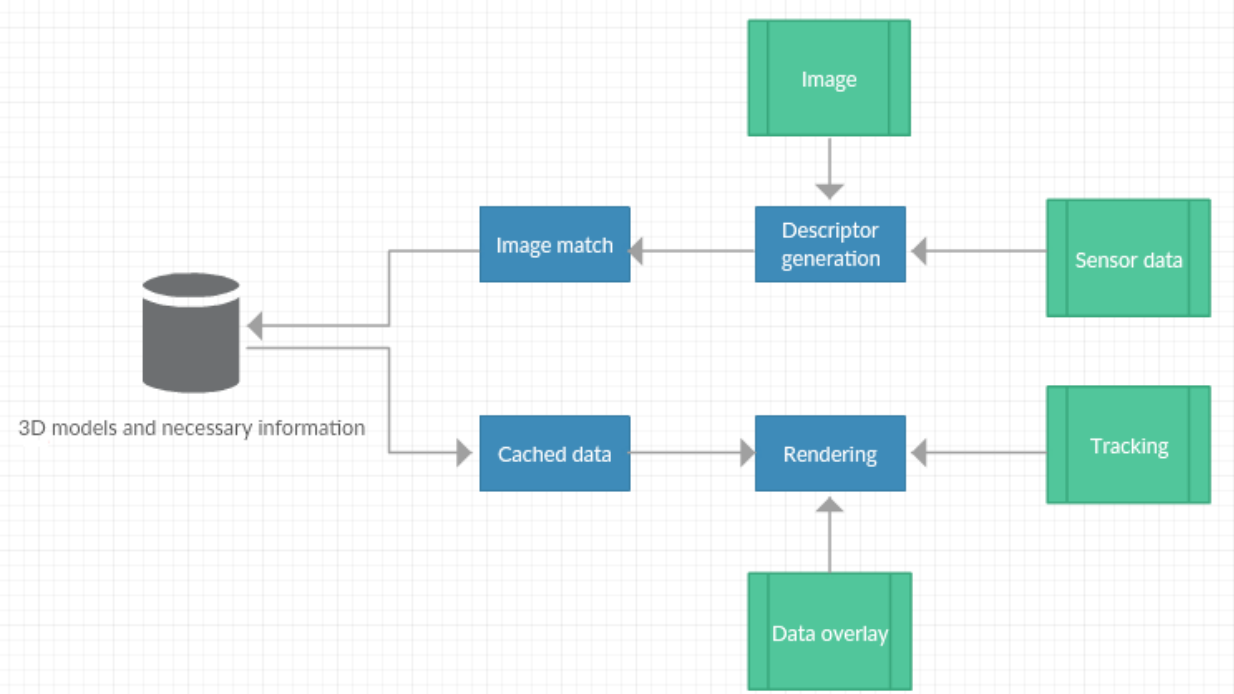

Figure 3: Virtual object generation process flow 
It takes 5 to 7 days to create one model. The entire project completion requires 500 to 700 hours. Scrum Product Backlog is shown in Figure 4.

\begin{tabular}{|c|c|c|}
\hline ID & V Task & V Priority 7 Duration Owner \\
\hline & 1 UX Design (Mock-Ups) & 8 Tamara \\
\hline & 2 Model specifications & 3 Talib \\
\hline & 3 User themes feature & 6 Lazar \\
\hline & 4 Database configuration & 4 Tamara \\
\hline & 5 UX Development & 10 Lazar \\
\hline & 6 Training and tests for users & 5 Talib \\
\hline
\end{tabular}

Figure 4: Product backlog

3. Prototype development

At this stage, a prototype application with basic functionality is created:

- display of the desired product in real space

- modification or removal of the product

- availability check

- the ordering process

4. Testing

Application testing involves running the application and allowing the app to use the camera of the device. Integrating wait period during which the application determines the position of the user based on the image analysis and defines the space for entering the 3D model. In the testing stage, it is important to monitor the position of the model and its positioning in the space. It is necessary to change the product and change the position in relation to the model.

\section{DEVELOPMENT OF AR SERVICES IN THE FURNITURE INDUSTRY}

The goal of the application is for the user and the application to clearly identify the object to which the multimedia content will be added. In this case, the selected element from the virtual catalog of furniture will be added. The main menu opens on the initial screen when the application starts.

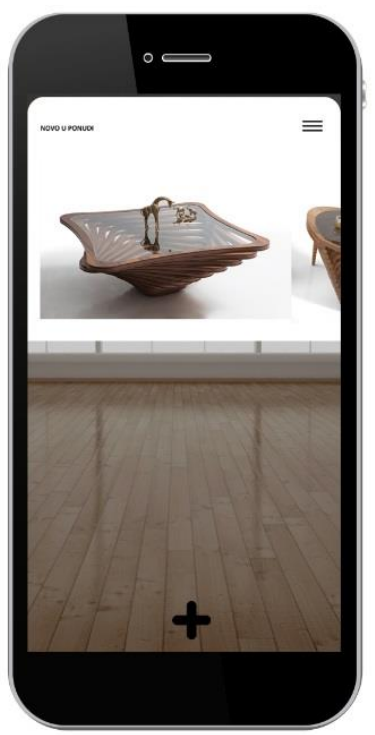

Figure 5: Main menu

Identification labels are assigned to real objects, based on previously analyzed system architecture.

There are several labeling techniques and the selection of technique depends on the properties of the real objects. Ideally, labels can be based on the natural characteristics of the real objects, usually visual, which makes the system simpler and cheaper.

The AR technology develops in two directions, depends on whether markers are used to initiate the search and display digital content:

- augmented reality based on markers

- augmented reality based on parameter reading

Augmented reality based on markers works by marker recognition using the reading device and igniting the process of transferring digital 
content from the database associated with the given label.

The developed application is based on reading the parameters received in many ways. AR application based on parameter reading collects data through various devices (GPS, sensors, compass) used for positioning the user. In accordance with the determined position, the selection and presentation of additional content is performed. Labels that are used to mark real objects in the augmented reality can be represented in the following categories:

- label based on a location

- relative label - relative to the space or positions of other objects nearby

- descriptive label

- label based on a picture of the real object

The label based on a location indicates the position and geographical orientation of the object in relation to the given coordinates:

- GPS label for geographical coordinates - geographic length, width, altitude

- compass label - geographical orientation for identification of the real object in a specific location,
- base station mobile telephony label can associate this data with real object thanks to the unique address and identification (Cell-ID) of each base station,

- Wi-Fi router label - uses the signal and unique MAC (Media Access Control) address of a router,

- Bluetooth device label - available for using via mobile device.

The application for mobile device retrieves an identification label in order to systematically process the request. In our case, it is furniture. Identification data can be: type of furniture, product dimensions and product prices.

AR implies that the process of identifying objects consists of the set of information related to the identification labels found in the database. The application overlaps pre-defined information labels that are in the database with the information about identification labels obtained from the reading. Our database contains 3D model of products and accompanying product information. An example of a 3D model is shown in Figure 5.
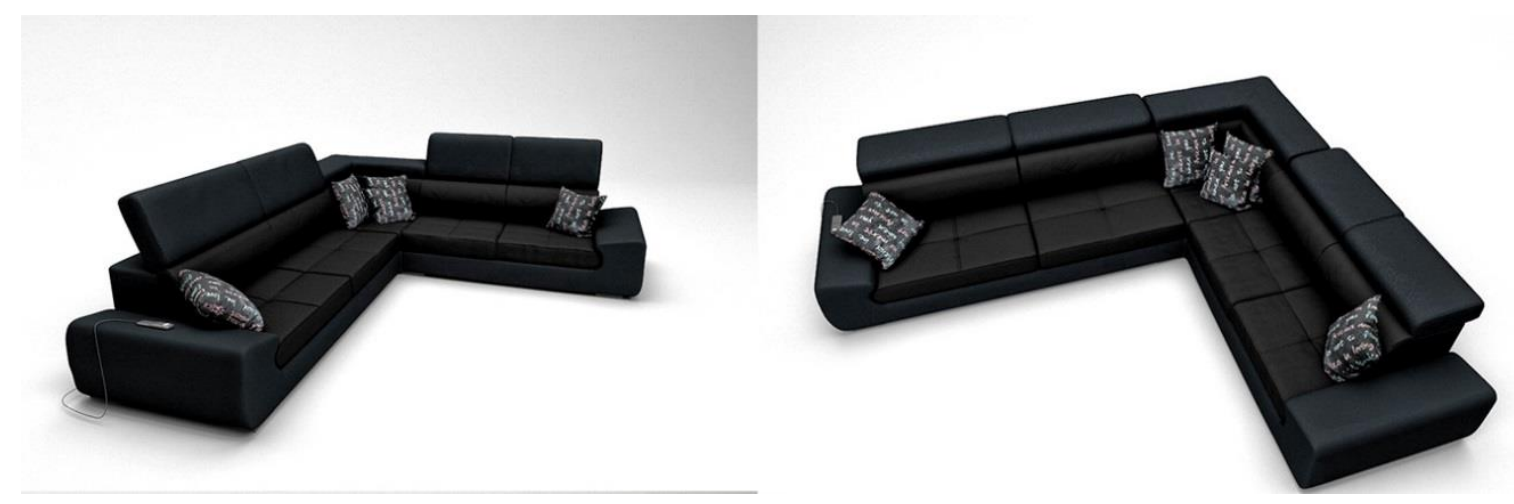

Figure 6: 3D model of product

Afterwards, the application uses the matching result to find the relevant contents that is attached to the identified real object, and then extends the viewing scene by superpositioning through the relevant medium. When the application completes the identification of the real object and retrieves the relevant contents, it uses different techniques in order to display the selected model incorporated with the real object.

The mathematical methods used in the second phase (Design) include:

- projective epipolar geometry

- geometric algebra

- rotation representation

- exponential maps 
- nonlinear optimization

- statistics.

For faster development of an application, there are various tools and SDKs, and the most useful ones are:

- Vuforia - one of the most useful software tools for developing the augmented reality for mobile devices (Android, Windows phone, iOS). This SDK was called QCAR

- Wikitude - the first SDK that provides JavaScript API that works with AR

- Argon - AR browser, based on HTML/JavaScript/CSS. Any web content with properly formatted metadata can be converted to an AR content

- ARToolKit - C multiplatform library successfully ported for platforms such as Android, Flash or Silverlight

In this case, the Vuforia software tool was used for identifying and positioning, as well as the $\mathrm{C \#}$ programming language for adding functionality.

\section{CONCLUSION}

$\mathrm{AR}$ and its application in the e-commerce is one of the current world trends. The goal of this paper is to indicate the possibility of improving efficiency and effectiveness of business by introducing the AR concept as a modern technology in e-commerce. In order to achieve the targeted goal, the field of e-commerce of the furniture manufacturing has been studied, analyzing the main advantages and disadvantages (Ronald, et al., 2001). An endeavor was made to present the importance of the mentioned technologies and their applications in business improvement and general performance of the company.

The goal of this paper was to develop a virtual catalog of products based on technologies of augmented reality. The possibilities of applying AR technology in the e-commerce of the company were pointed out through the realization of a practical example. So far, an initial version of the virtual catalog for iOS devices has been developed with several models of padded furniture. In the near future, the user interface will be upgraded, the catalog will be updated with the remaining models and positioning in the real space will be improved. Finally, AR catalog will be finalized with the feature of online shopping.

AR as technology will surely be more and more available and used in different fields and its application will facilitate and complement the various business processes and everyday life. The issues to be considered are whether it will continue to evolve equally or will it replace the virtual reality. Augmented reality is one of the technologies that can be used in achieving business advantage and is the type of technology that will improve business and contribute to user satisfaction with products and services.

\section{REFERENCES}

Ariso, J. M. (2017). Augmented Reality: Reflections on Its Contribution to Knowledge Formation. Berlin, Germany: De Gruyter.

Bogojević, P. (2017). Comparative Analysis of Agile Methods for Managing Software Projects, European Project Management Journal, 7(1), pp. 58-74.

Craig, A. (2013). Understanding Augmented Reality. Waltham, Massachusetts: Elsevier Inc.

Furht, B. (2011). Handbook of Augmented Reality. New York: Springer.

(2018). Global Augmented Reality (AR) Market Professional Survey Report. Dallas: Orbis Research.

Jung, T., \& Dieck, M. (2018). Augmented Reality and Virtual Reality: Empowering Human, Place and Business. Manchester: Springer International Publishing.

Kurubacak, G. A. (2017). Mobile Technologies and Augmented Reality in Open Education. Hershey, Pennsylvania: IGI Global.

Lee, I. (2009). Electronic Business: Concepts, Methodologies, Tools, and Applications. Hershey: IGI Global.

Ma, D., Gausemeier, J., Fan, X., \& Grafe, M. (2009). Virtual Reality \& Augmented Reality in Industry. Shanghai: Shanghai Jiao Tong University Press. 
Ma, M., Jain, L., \& Anderson, P. (2014). Virtual. Augmented Reality and Serious Games for Healthcare 1. Berlin: Springer.

Metz, R. (2012, August 2). Augmented Reality Is Finally Getting Real. Technology Review.

Morales, C. (2015). Develping Augmented Reality applications with Unity $3 D$ and Vuforia. Fowler: eAcademicBooks LLC.

Mullen, T. (2011). Prototyping Augmented Reality. (M. Barsolo, G. Schwartz, J. Nyquist, D. Zeidel, \& L. Welch, Eds.) Indianopolis, Indiana: John Wiley \& Sons Inc.

Patrick, S. (2017). The concise fintech compendium.

Paul, B.-D. (2004). eBusiness. Red Globe Press.

Peddie, J. (2017). Augmented Reality: Where We Will All Live. Tiburon: Springer International Publishing.
Radenković, B., Despotović-Zrakić, M., Bogdanović, Z., Barać, D., \& Labus, A. (2015). E-Business. Belgrade: Faculty of Organizational Sciences.

Rampolla, J., \& Kipper, G. (2012). Augmented Reality. Syngress.

Ronald, A., Baillot, Y., Behringer, R., Feiner, S., Julier, S., \& MacIntyre, B. (2001). Recent Advances in Augmented Reality. Computers \& Graphics, 15.

Tucker, O., Pierera, R., Shaw, L., \& Underwood, M. (2018). Augmented Reality and Virtual Reality in Healthcare Market Opportunities and Future Forecast to 2022 by Top Companies - Siemens Healthcare, CAE Healthcare, Layar B.V, Artificial Life. 